Measurement of effect of electron cyclotron heating in a tandem mirror plasma using a semiconductor detector array and an electrostatic energy analyzer

R. Minami, T. Imai, T. Kariya, T. Numakura, M. Uehara, K. Tsumura, Y. Ebashi, S. Kajino, Y. Endo, and Y. Nakashima

Citation: Rev. Sci. Instrum. 87, 11 E306 (2016); doi: 10.1063/1.4959574

View online: http://dx.doi.org/10.1063/1.4959574

View Table of Contents: http://aip.scitation.org/toc/rsi/87/11

Published by the American Institute of Physics

$\oplus$ SHIMADZU Powerful, Multi-functional UV-Vis-NIR and Excellence in Science FJis Spectjophotometers

Providing the utmost in sensitivity, accuracy and resolution for applications in materials characterization and science

- Photovoltaics

- Polymers

- Coatings

- Ceramics

- Thin films

- Inks

- DNA film structures

- Packaging materials

- Nanotechnology

- Paints

Click here for accurate, cost-effective laboratory solutions
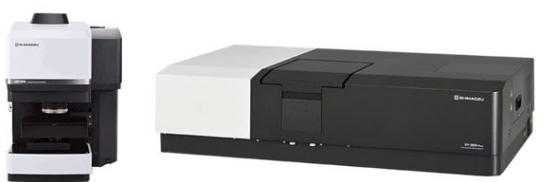

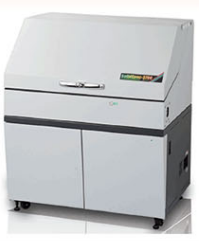




\title{
Measurement of effect of electron cyclotron heating in a tandem mirror plasma using a semiconductor detector array and an electrostatic energy analyzer
}

\author{
R. Minami, ${ }^{\text {a) }}$ T. Imai, T. Kariya, T. Numakura, M. Uehara, K. Tsumura, Y. Ebashi, S. Kajino, \\ Y. Endo, and Y. Nakashima \\ Plasma Research Center, University of Tsukuba, Tsukuba, Ibaraki 305-8577, Japan
}

(Presented 8 June 2016; received 3 June 2016; accepted 26 June 2016; published online 1 August 2016)

\begin{abstract}
Temporally and spatially resolved soft x-ray and end-loss-electron analyses of the electron cyclotron heated plasmas are carried out by using a semiconductor detector array and an electrostatic energy analyzer in the GAMMA 10 tandem mirror. The flux and the energy spectrum of the end loss electrons are measured by a multi-grid energy analyzer. Recently, the electron cyclotron heating power modulation experiments have been started in order to generate and control the high heat flux and to make the edge localized mode-like intermittent heat load pattern for the divertor simulation studies by the use of these detectors for electron properties. Published by AIP Publishing. [http://dx.doi.org/10.1063/1.4959574]
\end{abstract}

\section{INTRODUCTION}

The most practical application of the GAMMA 10 mirror plasma to the divertor simulator is to use the axial loss as the particle and heat flux simulator. ${ }^{1}$ One of the important high heat load divertor simulator experiments is the ELM (edge localized mode) study. By modulating the fundamental electron cyclotron heating $(\mathrm{ECH})$ at the plug region $(\mathrm{P}-\mathrm{ECH})$ power, we can obtain the arbitrary pulse heat load patterns. By changing the on/off timing, we can simulate the ELM intermittent heat pulses. One pulse heat load energy density depends on the ECH power.

In the GAMMA 10 tandem mirror, the P-ECH generates the axial ion confining potential. The $\mathrm{P}-\mathrm{ECH}$ drives a portion of the heated electrons into the loss cone and induces an intense axial flow of the warm electrons. Because the heating wave is injected from the high field side, only electrons moving toward the machine end couple with the heating wave. Each electron is perpendicularly accelerated or decelerated depending on its gyration phase. A portion of the axially flowing electrons is observed as the end loss electrons.

Open-ended magnetic fields produced for the magnetically confined plasma formation are still strong even in the outside regions of the coil locations. Plasma diagnostic systems including the $\mathrm{x}$-ray and charged particle detectors are placed in such high-field and limited narrow-spacing areas.

For the purpose of the above end-loss-electron observations, a semiconductor $\mathrm{x}$-ray detector (XSD) array and a multi-grid type electrostatic energy analyzer are employed so as to interpret the end loss physics. Semiconductor $\mathrm{x}-$ ray detector arrays are developed as position sensitive $\mathrm{x}$-ray imaging systems for the purpose of analyzing the temporally

Note: Contributed paper, published as part of the Proceedings of the 21st Topical Conference on High-Temperature Plasma Diagnostics, Madison, Wisconsin, USA, June 2016.

a)Electronic mail: minami@prc.tsukuba.ac.jp and spatially varying plasma behavior. Also, electrostatic analyzers of a multi-grid type have been used widely to measure the end loss particles from a mirror machine. The electrons flow into an end region along the lines of magnetic force. We directly detect the accelerated electrons at a machine end by making use of the open field configuration of GAMMA 10 and examining the effects of the $\mathrm{ECH}$ on electrons.

In this article, the measurement of electron cyclotron heated plasmas by a power modulation on the plug region and analysis of the high heat electron flux by the direct detection of the end loss electrons are reported.

\section{EXPERIMENTAL APPARATUS}

Plasma experiments have been carried out in GAMMA 10 , which is a minimum-B anchored tandem mirror with outboard axisymmetric plug and barrier cells in Fig. 1.

The plasma confinement is achieved by a magnetic mirror configuration as well as positive and negative potentials at the plug/barrier region formed by the $\mathrm{ECH}$. The main plasma confined in the central cell of GAMMA 10 is produced by ion cyclotron range of frequency (ICRF) wave. The ECH systems $(28 \mathrm{GHz}, 200 \mathrm{~kW}$ at barrier cells and $500 \mathrm{~kW}$ at plug and central cells) are prepared for producing the plasma-confining potentials in the plug and barrier regions, and also for direct electron heating in the central cell.

An x-ray diagnostic system using a 16-channel XSD array $^{2}$ is installed for analyzing the circularly shaped plug/barrier region plasmas. Figure 2(a) shows the schematic drawings of the temporally and spatially resolved $\mathrm{x}$-ray imaging system. The XSD system using a pinhole camera geometry includes the exchangeable photon absorber assembly. A XSD array has 16 channels for measurement of the plasma $\mathrm{x}$ ray radial profiles so as to make the $\mathrm{x}$-ray tomographic reconstructions. The XSD arrays are fabricated on p-type silicon wafer. The active area of each channel is $2 \times 5 \mathrm{~mm}^{2}$ and surface of the detector is covered with a $6 \mathrm{~nm} \mathrm{SiO}_{2}$ dead 


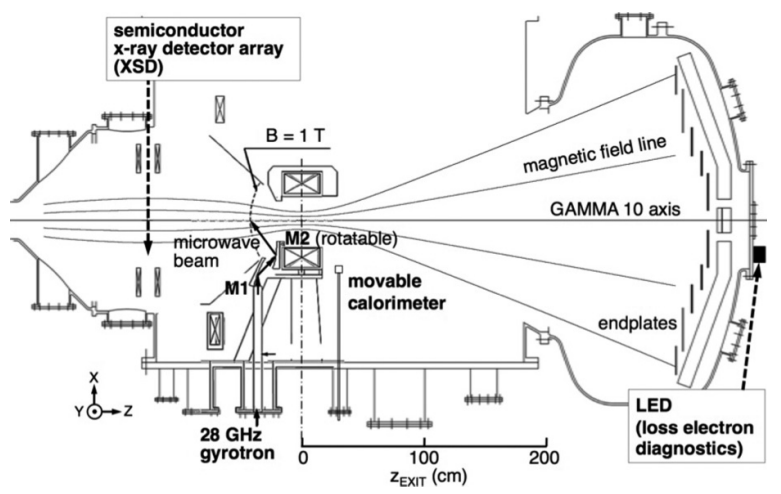

FIG. 1. Cross section of a plug/barrier and an end region in the GAMMA 10 tandem mirror. Microwave power is injected to the $1.0 \mathrm{~T}$ surface from the antenna. A multi-grid energy analyzer (LED) is installed behind the innermost endplate. An x-ray diagnostics system using a 16-channel XSD array is installed for circularly shaped barrier region plasma diagnostics.

layer. The x-ray absorption method is employed for measuring $T_{\mathrm{e}}$ using various parylene absorber films in front of the arrays.

The flux and the energy spectrum of the end loss electrons are measured by a multi-grid energy analyzer (loss electron diagnostics, LED) in Fig. 2(b). End loss electrons enter the analyzer through a small hole on an electrically floating end plate that is located in front of the end wall. The collector current of the analyzer corresponds to the electron current flowing into the end plate. With the LED we can obtain the energy distribution of loss particles up to about $10 \mathrm{keV}$. The energy selection is made by the negatively biased electron repeller grid in front of the collector, which is connected to machine ground through a low resistance to measure the electron current. The biasing voltage $\left(-V_{\mathrm{ER}}\right)$ of the electron repeller grid is swept during a shot. The LED is located at $r=30 \mathrm{~cm}$ behind the innermost end plate and is connected to the magnetic field line running $2.8 \mathrm{~cm}$ off the axis at the $1 \mathrm{~T}$ layer in the plug region.

The P-ECH system and locations of the diagnostic systems used in the preliminary P-ECH modulation experiment to generate the high and ELM-like heat flux are shown in Fig. 1. In the vacuum vessel, a launcher composed of an open ended corrugated waveguide and two mirrors (M1 and M2) is installed. It radiated the microwave power to the resonance layer as shown in Fig. 1. The microwave beam is obliquely injected into the resonance surface at an angle of $54^{\circ}$. The mirror M2 can be rotated around the horizontal axis and can change the beam direction.

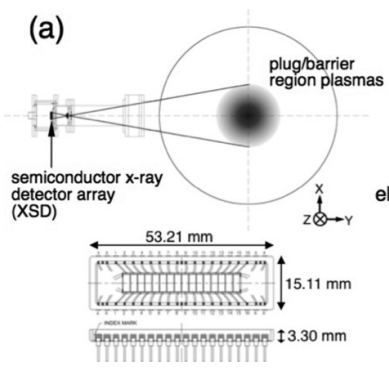

(b)

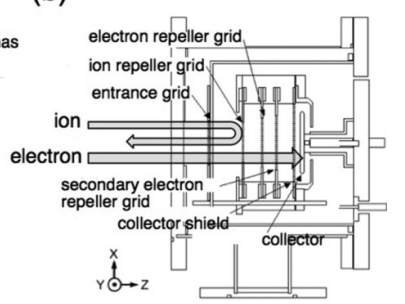

FIG. 2. Schematic drawings of (a) an x-ray diagnostics system using a 16 channel XSD array for barrier region diagnostics and (b) a multi-grid energy analyzer (loss electron diagnostics, LED).

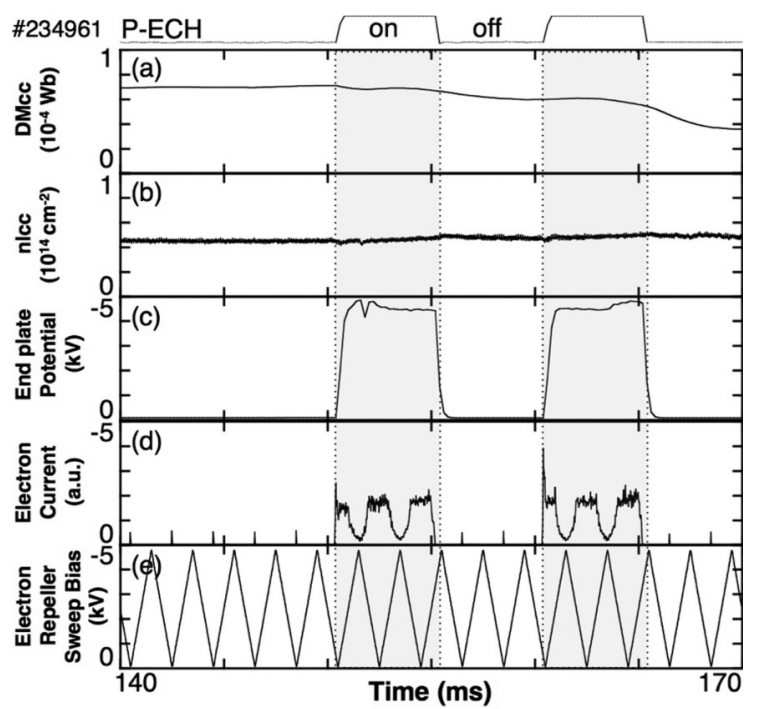

FIG. 3. Temporal evolution of (a) diamagnetism and (b) line density at the central-chord in the central cell. The end plate potential (c) at the west end. The end loss electron current (d) and the electron repeller sweep bias voltage (e) of the LED at the west end. The P-ECH power of about $380 \mathrm{~kW}$ is applied from $\mathrm{t}=151 \mathrm{~ms}$ to $171 \mathrm{~ms}$ with $100 \%, 100 \mathrm{~Hz}$ square wave power modulation.

\section{EXPERIMENTAL RESULTS}

The plasma experiment for the ECH power modulation is carried out by the use of a plasma discharge after the time of $t=140 \mathrm{~ms}$ (Fig. 3). During P-ECH from $t=151 \mathrm{~ms}$ to $171 \mathrm{~ms}$, the pulse of ECH power of about $380 \mathrm{~kW}$ for $5 \mathrm{~ms}$ is applied two times by $100 \%, 100 \mathrm{~Hz}$ square wave power modulation [gray region between dotted-lines in Fig. 3].

During P-ECH, a gradual decrease of the diamagnetism $D M_{\text {cc }}$ appears in Fig. 2(a) accompanied by a little increase of the line density $n l_{\mathrm{cc}}$ at the central-chord in the central cell [Figs. 3(a) and 3(b)]. The end plate potential is increased during P-ECH injection [Fig. 3(c)]. The end loss electron current is increased during P-ECH injection [Fig. 3(d)], which is measured with the LED at the west end. The pulse train of the electron current is due to sweep of the repeller voltage [Fig. 3(e)] for energy analysis. Its envelope represents the electron current.

The flux $I_{\mathrm{e}}$ flowing into the collector of the LED is plotted as a function of the electron repeller voltage $V_{\mathrm{ER}}$. Both the flux and the mean energy increase with increase in the P-ECH power, $P_{\mathrm{P}-\mathrm{ECH}}$. The energy spectra of the end loss electrons cannot be expressed with a single component Maxwellian, but they are well fitted to a two-component Maxwellian:

$$
I_{\mathrm{e}}=I_{\mathrm{L}} \exp \left(-\frac{V_{\mathrm{ER}}+\Phi_{\mathrm{EP}}}{T_{\mathrm{eL}}}\right)+I_{\mathrm{H}} \exp \left(-\frac{V_{\mathrm{ER}}+\Phi_{\mathrm{EP}}}{T_{\mathrm{eH}}}\right),
$$

for $V_{\mathrm{ER}} \geq\left|\Phi_{\mathrm{EP}}\right|\left(\Phi_{\mathrm{EP}}<0\right)$. Here, $T_{\mathrm{eL}}$ denotes the temperature of the bulk component and $T_{\mathrm{eH}}$ is that of the high energy tail component. The value of $I_{\mathrm{e}}$ is constant for $V_{\mathrm{ER}} \leq\left|\Phi_{\mathrm{EP}}\right|$ because the end plate effectively works as an electron repeller. With these parameters, an effective temperature $T_{\text {eff }}$ is defined as a measure of the mean energy:

$$
T_{\mathrm{eff}}=(1-\beta) T_{\mathrm{eL}}+\beta T_{\mathrm{eH}},
$$




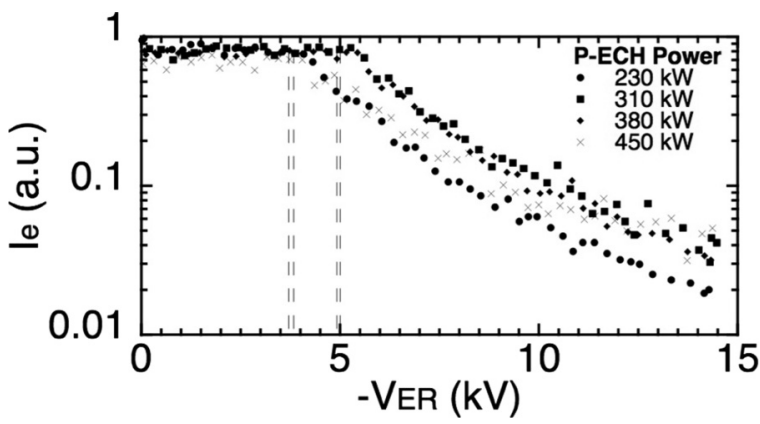

FIG. 4. Energy spectra of the end loss electrons measured with the LED for some $P_{\text {P-ECH }}(230,310,380$, and $450 \mathrm{~kW})$.

where $\beta$ is the flux fraction of the $T_{\mathrm{eH}}$ component to the total flux: $\beta=I_{\mathrm{H}} /\left(I_{\mathrm{L}}+I_{\mathrm{H}}\right)$. Here, $\beta$ is evaluated at $V_{\mathrm{ER}}=\left|\Phi_{\mathrm{EP}}\right|$. Typical energy distribution of electrons at some ECH powers is plotted in Fig. 4.

The dependence of $T_{\text {eff }}$ and the axis heat flow on $P_{\mathrm{P}-\mathrm{ECH}}$ is presented in Fig. 5. These values increase almost linearly with

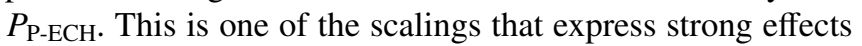
of the P-ECH.

The temporal evolution of the radial profiles of the lineintegrated $\mathrm{x}$-ray intensities (brightness) in the barrier region of GAMMA 10 is obtained during a single plasma shot. Figure 6 shows an overview of the temporal variation of the x-ray radial profiles obtained from the XSD with the $\mathrm{P}-\mathrm{ECH}$ power modulation. One can see a significant increase in the soft X-ray intensity due to the $\mathrm{ECH}$ in the plug region. The electron is strongly heated on the axis.

As one can see in the above examples, such plasmadiagnostics development will provide various findings of plasma-physics fundamentals, as well as applications including plasma control and stabilization.

These data indicate that the P-ECH is able to control the end loss ion and the electron fluxes by the use of power modulation, which can also produce the arbitrary heat load pattern like the various types of the ELM. The maximum

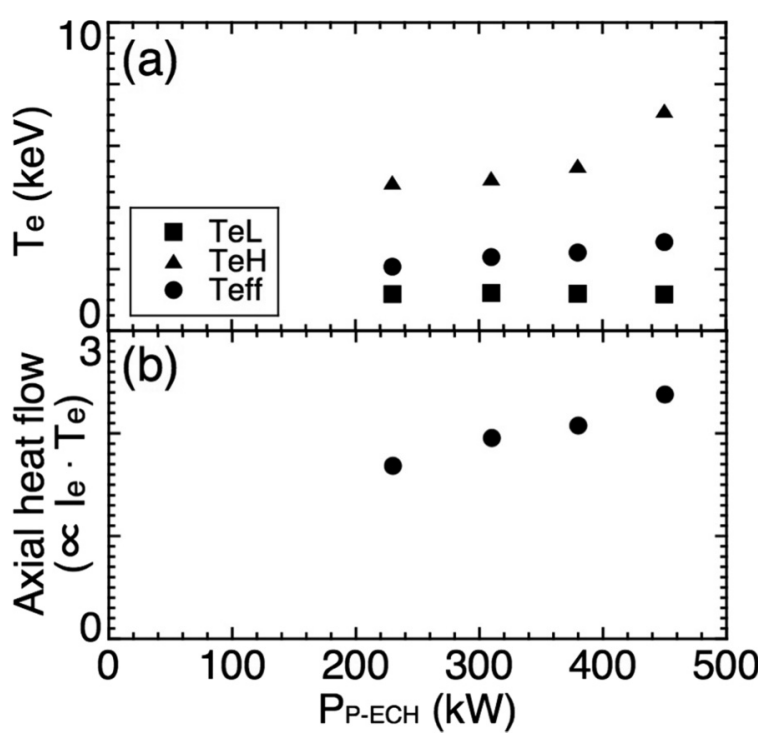

FIG. 5. (a) Electron temperature and (b) axis heat flow plotted as functions of the plug ECH power $P_{\mathrm{P}-\mathrm{ECH}}$

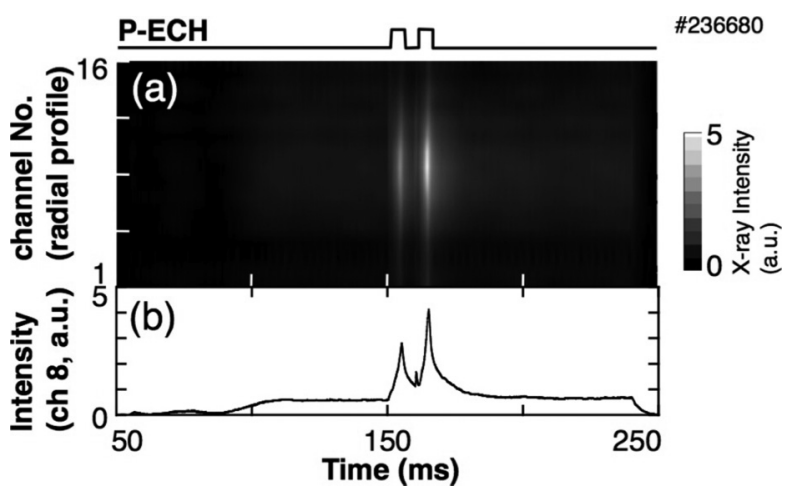

FIG. 6. (a) Temporal variation of the x-ray radial profiles in the barrier region obtained from the XSD array. White color region corresponds to the high $\mathrm{X}$-ray intensity region. (b) Temporal evolution of the soft X-ray intensity at the central-chord.

energy density obtained by using a movable calorimeter is over $0.7 \mathrm{MJ} / \mathrm{m}^{2} \mathrm{~s}^{1 / 2}$ with the pulse of $\mathrm{ECH}$ power of about $380 \mathrm{~kW}$ for $5 \mathrm{~ms}$. This value is still far lower than the ITER level, but it is expected to approach to the ITER level by the upgrade and combination of all the heating systems. ${ }^{3}$

\section{SUMMARY}

ECH power modulation experiments in GAMMA 10 have been started in order to generate and control the high heat flux for divertor simulation studies. $\mathrm{P}-\mathrm{ECH}$ is able to create the plug potentials and the electron flow. We have analyzed the characteristic features of the end loss electrons by using a semiconductor detector array and an electrostatic energy analyzer. The significant increment of soft x-ray intensity during a pulse of P-ECH operation has been obtained. From power scaling of electron flux in GAMMA 10, the higher $\mathrm{ECH}$ power generates the higher electron flux. The loss flux contains multi-components. Most of the loss flux is composed of warm electrons with energies from several hundred $\mathrm{eV}$ to a few keV. Until now, the maximum energy density obtained is over $0.7 \mathrm{MJ} / \mathrm{m}^{2} \mathrm{~s}^{1 / 2}$ with the pulse of $\mathrm{ECH}$ power of about $380 \mathrm{~kW}$ for $5 \mathrm{~ms}$.

\section{ACKNOWLEDGMENTS}

The authors thank the members of the GAMMA 10 group of the University of Tsukuba for their collaboration and valuable discussion during this study. This work is partially supported by the grant-in-aid for Scientific Research from Ministry of Education, Science, Sports and Culture of Japan (Grant No. 25249141) and National Institute for Fusion Science (NIFS) Collaborative Program (No. NIFS14KUGM090).

${ }^{1}$ T. Imai, M. Ichimura, Y. Nakashima, M. Sakamoto, I. Katanuma, M. Yoshikawa, T. Kariya, M. Hirata, J. Kohagura, R. Minami, T. Numakura, R. Ikezoe, K. Oki, K. Sakamoto, and GAMMA10 Group, Trans. Fusion Sci. Technol. 63(1T), 8 (2013).

${ }^{2}$ R. Minami, T. Imai, T. Kariya, T. Numakura, T. Eguchi, R. Kawarasaki, K. Nakazawa, T. Kato, F. Sato, H. Nanzai, M. Uehara, Y. Endo, and M. Ichimura, Rev. Sci. Instrum. 85, 11D807 (2014).

${ }^{3}$ T. Kariya, T. Imai, R. Minami, T. Numakura, T. Eguchi, T. Kato, Y. Endo, M. Ichimura, T. Shimozuma, S. Kubo, H. Takahashi, Y. Yoshimura, H. Igami, S. Ito, T. Mutoh, K. Sakamoto, H. Idei, H. Zushi, K. Nagasaki, F. Sano, M. Ono, and Y. Mitsunaka, Nucl. Fusion 55, 093009 (2015). 